\title{
Mental health care needs and preferences for mothers of children with a disability
}

Short title: Mental health of mothers

\author{
Authors \\ 1. Kim-Michelle Gilson, BSc (Hons), MPsych (Clinical), PhD \\ Post-Doctoral Fellow, Jack Brockhoff Child Health and Wellbeing Program, Centre \\ for Health Equity, Melbourne School of Global and Population Health, The \\ University of Melbourne, Australia; Level 5, 207 Bouverie St, Carlton VIC 3053; \\ kgilson@unimelb.edu.au; +61383449101
}

2. Elise Davis, BSc (Hons), $\mathrm{PhD}$

Associate Director, Jack Brockhoff Child Health and Wellbeing Program, Centre for Health Equity, Melbourne School of Global and Population Health, The University of Melbourne, Australia; Level 5, 207 Bouverie St, Carlton VIC 3053; eda@unimelb.edu.au; +61 383440921

3. Shae Johnson, BSc

Senior Consultant, Centre for Family Research and Evaluation, Drummond Street Services, 100 Drummond Street, Carlton Victoria 3053, shae.johnson@ ds.org.au; +61 396636733

This is the author manuscript accepted for publication and has undergone full peer review but has not been through the copyediting, typesetting, pagination and proofreading process, which may lead to differences between this version and the Version of Record. Please cite this article as doi: 10.1111/cch.12556

This article is protected by copyright. All rights reserved. 
4. Joan Gains

Research Assistant, Jack Brockhoff Child Health and Wellbeing Program, Centre for Health Equity, Melbourne School of Global and Population Health, The University of Melbourne, Australia; Level 5, 207 Bouverie St, Carlton VIC 3053;

gains_j@optusnet.com.au

\section{Dinah Reddihough, MD, BSc, FRACP, FAFRM}

Paediatrician; Developmental Disability and Rehabilitation Research, Murdoch Childrens Research Institute, Australia; Department of Paediatrics, The University of Melbourne, Australia; Department of Developmental Medicine, Royal Children's Hospital, Melbourne, Australia; Royal Children's Hospital, Flemington Road, Parkville, Victoria 3052; dinah.reddihough@rch.org.au

6. Katrina Williams, MBBS, MSc, PhD, FRACP, FAFPHM

Director, Department of Neurodevelopment \& Disability; APEX Australia Chair of Developmental Medicine, University of Melbourne; Honorary Research Fellow, Murdoch Childrens Research Institute; Royal Children's Hospital, 50 Flemington Road, Parkville, Victoria 3052; katrina.williams@ rch.org.au; +61 393455898 


\section{Acknowledgements}

We thank the mothers for their time and contribution to this study and the parent engagement group that advised on the research aims, study design and results.

This study was supported by a post-doctoral fellowship funded by the NHMRC Centre of Research Excellence in Cerebral Palsy (NHMRC APP1043013), an Early Career Grant from the University of Melbourne, Seed Funding from the State Trustees and support from the Jack Brockhoff Child Health and Wellbeing Program.

\section{Conflict of Interest}

The authors have stated that they had no interests that could be perceived as posing a conflict or bias. 


\section{Abstract}

Background: Mothers of children with a disability are at increased risk of poor mental health compared to mothers of typically developing children. The aim of the study was to describe the mental health care needs and preferences for support of mothers of children and young people aged 0-25 years with a disability.

Methods: A cross-sectional study, using an online survey with 294 mothers of children with a disability. Questions were asked about mental health, perceived need for support, barriers to accessing mental health care and preferences for support. Descriptive and chi-squared analyses were performed.

Results: High rates of mental ill health were self-identified in the previous 12 months, with reported clinically significant depression (44\%), anxiety (42\%), and suicidality (22\%). Nearly half $(48 \%)$ of the mothers reported high to very high psychological distress. While $75 \%$ of mothers perceived a need for professional support, only $58 \%$ attempted to access this. Key barriers to accessing support were caregiving duties making it difficult to schedule appointments (45\%) and not perceiving the mental health problem as serious enough to require help (36\%). Individual counselling was the preferred type of support $(66 \%)$ followed by professionally guided relaxation (49\%) and education about mental health (47\%). Support was considered most critical at the time of diagnosis and during medical intervention for their child.

Conclusions: Although mental health problems were common and mothers perceived the need for professional help, several key barriers were preventing mothers from accessing help. 
Our study suggests that improving mothers' knowledge of when and where to seek help (mental health literacy) may encourage their access to support. There also needs to be treatment that is accessible to mothers and therefore accommodates the high care demands that are placed upon them.

Keywords: Mental health, Disability, Mothers, Mental health care needs, Mental health services

\section{Key Messages (up to five on points of practice, policy or research)}

- Mothers of children with a disability report high rates of distress, depression, anxiety and suicidality.

- A high number of mothers do not access professional mental health support, despite perceiving a need for it.

- Individual counselling is the preferred type of support followed by other preventative strategies

- Mental health support is most wanted at the time of diagnosis and during their child's medical intervention. 


\section{Introduction}

Parents caring for children with a disability are at greater risk of poorer physical and psychological health than parents of children without disabilities (Singer 2006, DillonWallace, McDonagh et al. 2013, Gallagher \& Whiteley 2013) and experience higher rates of chronic stress, fatigue, chronic back pain and obesity, depression, as well as chronic sleep problems (Brehaut, Kohen et al. 2004, Raina, O'Donnell et al. 2005, Bourke-Taylor, Howie et al. 2012, Gallagher \& Hannigan 2014). Although there is an established body of research demonstrating that parents (particularly mothers) of children with a disability experience high rates of poor mental health, there is limited research on their anxiety, suicidality, and mental health service use and preferences.

\section{Maternal depression, anxiety and suicidality}

Research consistently shows that mothers of children with a disability have greater levels of depression compared to mothers of typically developing children, although the figures vary widely depending on the size of the sample and measure of depression (Singer 2006, Bailey, Golden et al. 2007). Limited research has examined clinical levels of anxiety in mothers of children with a disability, focusing mainly on measures of stress that combine depressive and anxiety symptom scores. This is despite anxiety being the most common mental health problem in Australia today (18\%; Australian Bureau of Statistics 2007). Maternal depression and anxiety can have deleterious consequences for the parenting role, family functioning and offspring wellbeing (Kahn, Brandt et al. 2004, White and Hastings 2004, Pilowsky, 
Wickramaratne et al. 2006). In the most severe cases, mothers may be forced to relinquish the care of their child (Victorian Equal Opportunity \& Human Rights Commission 2012).

Depression can also pose a significant risk for suicide (Brown, Beck et al. 2000). With no quantitative data on suicide in mothers of children with a disability there is a need to establish rates of suicidal behaviour, including ideation, plans and attempts in this vulnerable group of mothers.

\section{Self-perceived mental health}

There is little information about how mothers perceive their mental health overall and whether their self-perception is consistent with results from screening tools. Single item selfrated measures of mental health (SRMH) have been used as stand-alone indicators of mental health in large studies (Fleishman and Zuvekas 2007). Of interest, is whether mothers of children with a disability self-rate their mental health on a single item measure consistently with their responses on standardized multi-item measures. Given that mothers may be exposed to stress and challenges over many years, and have limited time to reflect on their own health, it may be difficult for them to recognise changes in their mental health and therefore disparity may occur between single item global measure scores and measures of anxiety and depression.

\section{Perceived need and access to professional mental health support}

Mothers of children with a disability often consider their health and wellbeing to be of a low priority and are reluctant to use services for their own needs, especially in the context of low 
household income and prioritisation of funds for their child's care needs (Beresford, 1994; Sloper, 1999). However, very little research has examined whether mothers perceive a need for professional mental health support, whether they access this support and the barriers they face in obtaining it. Understanding the proportion of mothers who perceive a need for mental health support and go on to access support and treatment is important given that delays in seeking effective help for mental health problems can lead to poorer outcomes (Stephenson 2000, Conus and McGorry 2002). For mothers of children with a disability, it is likely that accessing help will be difficult given that they have a lack of time, financial constraints and often prioritise their child's care needs over their own (Bourke-Taylor, Pallant et al. 2012). In addition to understanding the barriers to accessing mental health support, mothers' treatment preferences and the timepoints that they consider support to be most needed is important to inform new approaches to better supporting the mental health of mothers with a child with disabilities.

\section{Study aims}

The aims of this study were to report the broad mental health status of mothers (including anxiety, depression, psychological distress and suicidality) and investigate their help-seeking behaviour. Perceived need, access and barriers to professional mental health support, preferences for mental health interventions and the timing of when support is most needed were examined. Additionally, this study aimed to examine mental health and help seeking across subgroups of the single item of SRMH.

\section{Method}




\section{Participants}

Participants were recruited through a flyer that invited all mothers of children with a disability to participate in a research study on mental health. Both the flyer and participant information sheet emphasised that the study was open to all mothers of children with a disability. The flyer was disseminated through disability services across Victoria, Australia by newsletters, social media and websites. To be eligible for inclusion, mothers had to have a child or young person with a disability aged 0-25 years and be aged 18 years or older.

\section{Survey questionnaire}

Prior to distributing the survey, the questionnaire and methodology was discussed with a parent and carer engagement group from the University of Melbourne, which includes six mothers who guide and provide advice about research. This study used an online survey that was approved by the ethics review board at the University of Melbourne, Australia (HREC 1543687.3). Informed consent was obtained from the mothers by requesting that they read an online consent form, tick their agreement and then proceed to the survey. All mothers' responses were anonymous.

\section{Measures}

Demographic information including age, country of birth, marital status, education, employment, and income were collected. Information about mental health, help seeking, and preferences for mental health support were collected. 


\section{Mental health}

Anxiety. The Generalized Anxiety Disorder Scale (GAD-7; Spitzer, Kroenke et al. 2006) is a 7-item self-report to assess anxiety symptoms and screen for generalized anxiety disorder. Scores range from 0 to 21 . Cut points of 5, 10, and 15 represent mild, moderate, and severe levels of anxiety, respectively. The GAD-7 has demonstrated good internal consistency $(\alpha=$ .92 ) and test-retest reliability (intraclass correlation $=0.83$ ). Scores on the measure also evidenced good procedural validity; the GAD-7 self-report scores and GAD-7 scores derived from a clinical interview administered by a mental health professional produced similar results.

Depression. The Beck Depression Inventory-II (BDI-II; Beck, Steer et al. 1996) is a 21-item self-report measure of the severity of depressive symptoms in areas such as mood, pessimism, sense of failure, and somatic symptoms. There is evidence for the reliability $(\alpha=$ .92), validity, and utility of the instrument (Dozois, Dobson et al. 1998). Interpretive ranges for scores are: 0-13 minimal depression; 14-19 mild depression; 20-28 moderate depression; and 29-63 severe depression.

Psychological distress. The Kessler Psychological Distress Scale (K-10) is a 10-item selfreport measure of psychological distress. Scores for each item range from 0 (no distress) to 5 (highly distressed), with total scores ranging from 0 to 50 . The scale possesses excellent internal consistency, with a Cronbach alpha $(\alpha)$ of 0.93 (Fassaert, De Wit et al. 2009). Established cut-off scores used in this study are adopted from those described in a national epidemiological study (Australian Bureau of Statistics 2007). These are: 0-15 no distress; 
16-21 moderate distress; 22-29 high distress; and 30-50 very high distress.

Single-item measure of self-rated mental health (SRMH). The item asks respondents to rate their mental health on a five-point scale from excellent to poor.

Suicidality. Three items based on the National Survey of Mental Health and Wellbeing (Australian Bureau of Statistics 2007) were used to examine suicidality in the previous 12 months (thoughts, plan and attempt).

Mothers were also asked whether they had received a previous diagnosis of a mental health disorder (yes/no) and whether they were currently on prescribed medication for anxiety or depression (yes/no).

\section{Help seeking}

Perceived need and service access. Perceived need was measured by asking mothers if in the last year there was a time when they thought they needed support from a professional for their mental health. Access was measured by asking if they had accessed support from a professional. Mothers who indicated 'yes' were asked to indicate the professional from whom they had received this support. These items were adapted from similar surveys (Ho, Fung et al. 2008, Pagura, Fotti et al. 2009, Wong, Lam et al. 2010). An additional question on the helpfulness of this support was included, using a 5 point scale from $1=$ Not at all helpful to 5 $=$ Extremely helpful. Knowledge of services was explored with a single item using a 5 point scale from 1 = No knowledge to $5=$ Very knowledgeable. 
Barriers to accessing support. If mothers perceived a need for mental health care but had not accessed support, they were asked to select the main barriers from a selection of 13 possible options. Indirect (belief that the problem cannot be helped) and direct (lack of available services or waiting lists) barriers were included, adapted from similar research (Owens, Hoagwood et al. 2002, Australian Bureau of Statistics 2007).

\section{Mental health support}

Eleven types of evidence based mental health interventions was assessed, with response options from 1 = would not consider to $3=$ definitely consider. Support options were based on treatment preferences within previous surveys on mental health (Lang, 2005; Eisenberg, Golberstein, \& Gollust, 2005; Riedel-Heller, Matschinger \& Angermeyer, 2005) and included group, individual and web based formats.

\section{Timing of mental health support}

One question asked participants how much they thought professional support for their mental health was needed at various time points using a five-point Likert scare ( $1=$ extremely needed to $5=$ Not at all needed). A second question asked if they had ever received professional support for their mental health at each time point (Yes/No/Can't remember/Not applicable).

\section{Data Analysis}

Given the lack of existing data in this area, this paper primarily takes a descriptive approach.

Chi-square tests were used to determine whether SRMH groups differ on key variables of 
interest. Post hoc analysis was conducted using a z-test of two proportions. SPSS Version 22 was used.

\section{Results}

\section{Characteristics}

The survey was completed by 294 mothers resident in Australia, of which $89 \%$ resided in Victoria. The participant characteristics are displayed in Table 1. Comparisons were made to population data using ABS Census data (Australia Bureau of Statistics, 2011), restricted to females, age 25 to 54 years and residing within the state of Victoria. For education, $78 \%$ of study participants reported completing higher education compared to $61 \%$ of the general population and for employment, 53\% of participants were in the labour force compared to $73 \%$ of the general population. Using total household income for families, $23 \%$ of study participants reported less than $\$ 40,000$ for the annual household income compared to $20 \%$ of the general population.

The 294 mothers had a total of 376 children with disabilities, with $78 \%$ having one child, $16 \%$ with 2 children and $6 \%$ with 3 or more children with disabilities. The average age of the children was 11.2 years (SD 4.9). The reported severity of the disabilities were mild (29\%), moderate $(47 \%)$ and severe $(24 \%)$. The most commonly reported childhood disability types were autism spectrum disorder (ASD) (57\%), intellectual disability (26\%), developmental delay (23\%), and cerebral palsy (28\%). Comorbidity was common, with $57 \%$ of children having two or more disabilities. 


\section{INSERT TABLE 1 HERE}

\section{Mental health}

Table 2 displays the mental health characteristics of mothers; $44 \%$ exceeded depression clinical cut-off points on the BDI-II, $42 \%$ showed clinically significant anxiety scores on the GAD-7, 31\% reported high psychological distress and 17\% reported very high distress on the K10. Overall suicidality (ideation, plan or attempt) in the previous 12-months was reported by $22 \%$ of mothers and $4 \%$ of the overall sample reported a past suicide attempt. Percent of anxiety and depression in the four main disability groups was also estimated. Fifty percent of mothers with children with ASD reported depression and anxiety. These figures were 53\% and $55 \%$ for mothers of children with intellectual disability, $45 \%$ and $46 \%$ for mothers of children with developmental delay and $36 \%$ and $35 \%$ for mothers of children with cerebral palsy.

\section{INSERT TABLE 2 HERE}

\section{Perceived need, unmet need and access to mental health support}

Overall $75 \%(\mathrm{n}=222)$ of mothers perceived a need for professional help over the past 12 months, of whom 58\% $(n=128)$ accessed professional mental health support. Therefore $42 \%$ $(\mathrm{n}=94)$ did not access professional support despite having a perceived need. In mothers with clinical levels of anxiety $(n=124)$ and depression $(n=128), 91 \%$ perceived a need for professional help and $47 \%$ of those with depression and $51 \%$ of those with anxiety did not go on to access support. In the mothers who had accessed mental health services in the previous 
12 months $(n=128)$, the professional most accessed was a psychologist or psychiatrist $(73 \%)$ or their GP (68\%) and $21 \%$ consulted a social worker or counsellor. Of the professional support accessed, $62 \%$ rated it as extremely/very helpful.

\section{Comparisons across self-rated mental health groups}

Chi-square and post hoc z-tests indicated that there was a significantly greater proportion of mothers with anxiety, perceived need for mental health treatment, and previous mental illness in the 'poor' and 'fair' SRMH group compared to the 'good' and 'very good/excellent' SRMH group, $\mathrm{p}<.05$ (no significant difference was observed between 'poor' and 'fair' SRMH groups). As shown on Table 3 the proportion of mothers having depression in the 'poor' SRMH group was significantly greater than all other SRMH groups, $p>.05$. There were no statistically significant differences in proportions of mothers between the SRMH groups who accessed mental health support.

In mothers in the 'good' SRMH group, 76\% reported a perceived need for mental health support and nearly a third reported clinical levels of anxiety (31\%) or depression (30\%). The proportion of mothers having anxiety, depression, perceived need for mental health treatment, and previous mental illness in the 'good' SRMH group was significantly greater than in the 'very good/excellent' SRMH group $\mathrm{p}<.05$. Perceived need was not statistically significantly different between the 'good' and 'fair' SRMH group.

\section{INSERT TABLE 3 HERE}

\section{Barriers to accessing support}


The main barriers to accessing support reported by mothers were that caring responsibilities made it hard to schedule appointments (45\%), not perceiving the mental health problem as severe or serious enough to require help (36\%), preference for managing themselves (31\%) and thinking that nothing could help (28\%). These barriers were also the most endorsed in mothers reporting clinical levels of depression or anxiety (data not shown).

\section{Preferences for support}

Table 4 shows the preferences for support. Activities that mothers were most willing to consider for their mental health were individual counselling (66\%), guided relaxation programs facilitated by a health professional (49\%), education about mental health and available supports (47\%), guided exercise programs (46\%) and relationship support (32\%). These five activities were also the most endorsed in mothers who reported clinical levels depression or anxiety (data not shown).

\section{INSERT TABLE 4 HERE}

\section{Timing of mental health support}

The proportion of mothers who reported the need for professional mental health support at the specific time points were: child's diagnosis (88\%), during acute illness or medical intervention for their child (84\%), changes to do with schools (74\%), during key developmental changes $(76 \%)$, when changing major service providers $(54 \%)$, when a crisis was over (50\%) and when there was a major family change (22\%). Only $24 \%$ received mental health support at the time of diagnosis, $22 \%$ received it at the time of serious illness or 
medical intervention for their child, $25 \%$ when changes to do with schools occurred, $12 \%$ when going through key developmental changes, $10 \%$ when changing major service providers, $20 \%$ when a crisis was over, and $22 \%$ during major family change.

\section{Discussion}

Mothers of children with a disability in this study reported high rates of distress, depression, anxiety and suicidality. There was a high perceived need for professional mental health support, which many did not access. Mothers reported common barriers that prevented then accessing support and there was a strong preference for individual counselling. Mothers wanted mental health support at the time of their child's diagnosis and medical intervention. These findings provide directions for supporting mothers' mental health.

\section{Prevalence of mental health problems}

The prevalence of clinically significant depression and anxiety for mothers in this study was substantially higher than the prevalence of depression and anxiety for women in the general Australian population (5\% and 18\% respectively) (Australian Bureau of Statistics 2007). Furthermore, suicidality rates were significantly higher in this sample of mothers compared to those reported for females in the general Australian population (22\% versus $3 \%)$ and for attempted suicide ( $4 \%$ versus $0.5 \%$ ). The high proportion of suicidality is not surprising given the poor mental health in our sample, and that suicidality is greatest in those with mental illness (Australian Bureau of Statistics 2007). These results support the need for prevention and early intervention strategies targeting anxiety, depression and suicidiality in 
mothers of children with a disability. Our study found that most mothers had a strong preference for individual counselling, with a smaller proportion considering options that could be preventative or provided at low cost such as education, professionally guided relaxation and exercise programs.

Our results are consistent with those reported in the existing literature. For example in a sample of 99 mothers of children with intellectual disability in Pakistan, 35\% met criteria for anxiety and $45 \%$ for depression (Azeem et al., 2013). Furthermore, in Sweden, Olsson and Hwang (2001) found $45 \%$ of mothers of children with intellectual disability and $50 \%$ of mothers of children with ASD had depression. However, it must be recognised that our methodology, using an internet survey and advertising for a study on mental health, could introduce selection bias with respondents who have a particular interest in mental health or have had negative experiences with their mental health being over sampled. This, as well as our sample comprising a high proportion of mothers of children with ASD (57\%), indicates our results may not be generalisableto the broader population of mothers with children who have a disability.

\section{Barriers to accessing services}

While $75 \%$ of mothers perceived a need for support, $42 \%$ of those had not attempted to access this. The main reported barrier to accessing support was caregiving responsibilities that made it difficult to schedule appointments. More accessible forms of treatment are needed for mothers of children with a disability given their ongoing full-time care demands. This may include the provision of short-term respite for the child so that they have the time to 
access therapy. Alternatively, internet interventions can offer a non-threatening avenue to psychological help, be completed at times suitable for the mother and are a low cost alternative. Importantly, there is growing evidence for the efficacy of online interventions for mental health conditions such as depression compared to traditional face-to-face treatment delivery (Andrews, Cuijpers et al. 2010). However, our results suggested that online therapeutic interventions are less favoured $(25 \%)$ by parents, which warrants further investigation.

Three further barriers to accessing mental health support were the belief that the problem was not severe enough, preferring to manage alone and thinking that nothing could help. These results indicate the need to increase mothers' knowledge about mental health problems and benefits of mental health treatment and available support, especially as only $50 \%$ were confident in their knowledge of support options. This approach, consistent with mental health literacy, involves the knowledge and beliefs about mental health disorders, which aid their recognition, management or prevention (Jorm 2000). Providing education is a potentially low cost preventative approach that would enhance mental health literacy.

\section{Self-reported mental health and perceived need}

The proportion of mothers who reported accessing professional mental health support did not significantly differ across self-rated mental health groups amongst those who perceived a need for it. This was despite a clear, expected trend for anxiety, depression, suicidality and perceived need for support. This suggests that factors other than mental health severity influence accessing support. 
Another key finding was that some mothers who reported their mental health as 'good' also reported a perceived need for mental health support (76\%) and this was not significantly different to the proportion of mothers in the 'fair' group. Furthermore, a moderate proportion of mothers who reported their mental health as good, reported clinical levels of anxiety (30\%) and depression (31\%). One explanation could be that mothers emotions have been 'normalised' within their caregiver role and, has led mothers with moderate mental health needs to overestimate their mental health. There were also very few significant differences between the 'poor' and 'fair' groups on mental health and help seeking variables. These findings suggests that the single item self report of mental health will not be sufficient to assess whether there are clinical mental health needs and a perceived service need for treatment.

\section{Limitations}

There are several limitations that must be considered when interpreting the results.

Firstly, selection bias, as discussed above, is a risk given the online nature of the survey. Access to patient health datasets to explore diagnostic and mental health service use of parent carers of children and young people with disabilities would address recruitment-related bias in this study. However, it must be noted that a considerable number of mothers also responded that did not report difficulties with their mental health.

Secondly, as also discussed above, mothers of children with ASD were oversampled (57\%). Research has found that mothers of children with ASD have significantly poorer mental health than mothers of children with other developmental disabilities (Carter, Martinez-Pedraza Fde et al. 2009, Bourke-Taylor, Pallant et al. 2012). Statistical analysis on 
mental health variables across all disability groups was not possible because of very small numbers in some disability groups (e.g. Down syndrome). However, basic descriptive analyses in the most recruited disability groups (developmental delay, ASD and intellectual disability) estimated similar between group levels of anxiety and depression. Future quantitative research is needed that is sufficiently powered to allow comparisons on maternal mental health differences for different child disabilities, including a comparison group of mothers of children without disabilities.

A third limitation is the lack of independent validation of the accuracy of self-report data on mental health and service utilisation. The study employed self-report measures of depression and anxiety symptomatology rather than diagnostic instruments, and specific barriers and support options were presented that may not have captured mothers' preferences.

A final limitation lies with the use of multiple comparisons across the subgroups of the single-item measure of self-reported mental health. This may have increased the risk of committing a Type 1 error, potentially identifying incorrect significant differences.

\section{Conclusion}

In this study, a high proportion of mothers reported anxiety, depression and suicidality. Common barriers were reported that prevented mothers from accessing professional mental health support. Improving mothers' knowledge of when and where to seek help (mental health literacy) may increase their access of this support. Changes in the way services are delivered, either for mothers or their children are also needed so they can access individual counselling even though they have high child-care demands. 
This article is protected by copyright. All rights reserved. 


\section{References}

Andrews, G., et al. (2010). "Computer therapy for the anxiety and depressive disorders is effective, acceptable and practical health care: a meta-analysis." PLoS One 5(10): e13196.

Australia Bureau of Statistics (2011). Census Basic Community Profiles (cat.no. 2001.0), Australian Bureau of Statistics.

Australian Bureau of Statistics (2007). National Survey of Mental Health and Wellbeing: Summary of Results. Canberra, Australian Bureau of Statistics.

Bailey, D. B., Jr., et al. (2007). "Maternal depression and developmental disability: research critique." Ment Retard Dev Disabil Res Rev 13(4): 321-329.

Beck, A. T., et al. (1996). Manual for the Beck Depression Inventory-II. San Antonio, TX, Psychological Corporation.

Bourke-Taylor, H., et al. (2012). "Self-reported mental health of mothers with a school-aged child with a disability in Victoria: a mixed method study." J Paediatr Child Health 48(2): 153-159.

Bourke-Taylor, H., et al. (2012). "Predicting mental health among mothers of school-aged children with developmental disabilities: The relative contribution of child, maternal and environmental factors." Research in Developmental Disabilities 33(6): 1732-1740.

Bourke-Taylor, H., et al. (2012). "Predicting mental health among mothers of school-aged children with developmental disabilities: the relative contribution of child, maternal and environmental factors." Res Dev Disabil 33(6): 1732-1740.

Brehaut, J. C., et al. (2004). "The Health of Primary Caregivers of Children With Cerebral Palsy: How Does It Compare With That of Other Canadian Caregivers?" Pediatrics 114(2): e182-e191.

Brown, G. K., et al. (2000). "Risk factors for suicide in psychiatric outpatients: A 20-year prospective study." Journal of Consulting and Clinical Psychology 68(3): 371-377.

Carter, A. S., et al. (2009). "Stability and individual change in depressive symptoms among mothers raising young children with ASD: maternal and child correlates." J Clin Psychol 65(12): 1270-1280.

Conus, P. and P. D. McGorry (2002). "First-episode mania: a neglected priority for early intervention." Australian and New Zealand Journal of Psychiatry 36(2): 158-172. 
Dillon-Wallace, J. A., et al. (2013). "How Stable is the Well-Being of Australian Mothers Who Care for Young Children with Special Health Care Needs?" Journal of Child and Family Studies 23(7): 1215-1226.

Dozois, D. J. A., et al. (1998). "A psychometric evaluation of the Beck Depression InventoryII." Psychological Assessment 10(2): 83-89.

Fassaert, T., et al. (2009). "Psychometric properties of an interviewer-administered version of the Kessler Psychological Distress scale (K10) among Dutch, Moroccan and Turkish respondents." Int J Methods Psychiatr Res 18(3): 159-168.

Fleishman, J. A. and S. H. Zuvekas (2007). "Global self-rated mental health: associations with other mental health measures and with role functioning." Medical Care 45(7): 602-609.

Gallagher, S. and A. Hannigan (2014). "Child problem behaviours are associated with obesity in parents caring for children with developmental disabilities." Res Dev Disabil 36C: 358365.

Gallagher, S. and J. Whiteley (2013). "The association between stress and physical health in parents caring for children with intellectual disabilities is moderated by children's challenging behaviours." J Health Psychol 18(9): 1220-1231.

Graungaard, A. H., et al. (2011). "When resources get sparse: a longitudinal, qualitative study of emotions, coping and resource-creation when parenting a young child with severe disabilities." Health (London) 15(2): 115-136.

Ho, S. M. Y., et al. (2008). "Overprotection and the psychological states of cerebral palsy patients and their caretakers in Hong Kong: a preliminary report." Hong Kong Medical Journal 14(4): 286-291.

Jorm, A. F. (2000). "Mental health literacy: Public knowledge and beliefs about mental disorders." The British Journal of Psychiatry 177(5): 396-401.

Kahn, R. S., et al. (2004). "Combined effect of mothers' and fathers' mental health symptoms on children's behavioral and emotional well-being." Archives of Pediatrics \& Adolescent Medicine 158(8): 721-729.

Mechanic, D. (1966). "The Sociology of Medicine: Viewpoints and Perspectives." Journal of Health and Human Behavior 7(4): 237.

Owens, P. L., et al. (2002). "Barriers to children's mental health services." Journal of the American Academy of Child \& Adolescent Psychiatry 41(6): 731-738. 
Pagura, J., et al. (2009). "Help Seeking and Perceived Need for Mental Health Care Among Individuals in Canada With Suicidal Behaviors." Psychiatric Services 60(7): 943-949.

Pilowsky, D. J., et al. (2006). "Family discord, parental depression, and psychopathology in offspring: 20-year follow-up." J Am Acad Child Adolesc Psychiatry 45(4): 452-460.

Raina, P., et al. (2005). "The health and well-being of caregivers of children with cerebral palsy." Pediatrics 115(6): e626-636.

Singer, G. H. S. (2006). "Meta-analysis of comparative studies of depression in mothers of children with and without developmental disabilities." American Journal on Mental Retardation 111(3): 155-169.

Spitzer, R. L., et al. (2006). "A brief measure for assessing generalized anxiety disorder: the GAD-7." Archives of Internal Medicine 166(10): 1092-1097.

Stephenson, J. (2000). "Delay in Treating Schizophrenia May Narrow Therapeutic Window of Opportunity." JAMA 283(16): 2091.

Victorian Equal Opportunity \& Human Rights Commission (2012). Desperate Measures: The relinquishment of children with disability into state care in Victoria, Victorian Equal Opportunity \& Human Rights Commission.

White, N. and R. P. Hastings (2004). "Social and Professional Support for Parents of Adolescents with Severe Intellectual Disabilities." Journal of Applied Research in Intellectual Disabilities 17(3): 181-190.

Wong, F. K., et al. (2010). "Depression literacy among Australians of Chinese-speaking background in Melbourne, Australia." BMC Psychiatry 10: 7. 
Table 1. Characteristics of Mothers of Children with a Disability $(\mathrm{N}=294)$

\section{Socio-demographics}

n $(\%)$

Age

18-24 years

$25-34$

35 (12)

$35-44$

$136(46)$

$45-54$

107 (36)

$55+$

11 (4)

Relationship status

Married/De facto

231 (79)

Separated/divorced

$41(14)$

Widowed

3 (1)

Single

18 (6)

Born in Australia

244 (83)

Aboriginal or Torres Strait Islander Origin

$26(9)$

Employment status

Full-time/part time/casual

Full-time carer

$75(25)$

Not in the labour force

$47(16)$

Highest level of education

Year 10 or equivalent

Year 12 or equivalent

$26(9)$

Undergraduate/Diploma/Certificate/Graduate

$229(78)$

Healthcare card holder

Child

$180(61)$

Family

86 (29)

Not eligible

$23(8)$

Household income

Annual household income less than or equal to $\$ 40,000$

$66(23)$

This article is protected by copyright. All rights reserved. 
This article is protected by copyright. All rights reserved. 
Table 2. Mental health, perceived need and access to support $(\mathrm{N}=294)$

\section{Variable}

Mean Depression (BDI-II)

Clinically significant/depressive disorder (score >20)

Severe depression (score $>29$ )

Mean Anxiety (GAD-7)

Clinically significant/ anxiety disorder (score $\geq 10$ )

Severe anxiety (score $\geq 15$ )

Comorbid clinically significant Anxiety and Depression

Mean Psychological distress (K10)

Low (score 10-15)

Moderate (score 16-21)

High distress (score 22-29)

Very high distress (score 30-50)

Perceived need for professional mental health support

Accessed support previous 12 months?

Knowledge of mental health services to access

Very knowledgeable/knowledgeable

Some knowledge

No knowledge

GP use for own health (yes)

Previous mental ill health diagnosis (yes)

Psychotropic medication for anxiety or depression (yes)

Suicidality

Ideation, plan or attempt in previous 12 months (- yes)

Attempt only (- yes)

\section{n $(\%)$ or Mean (SD)}

$18.5(11)$

128 (44)

$54(18)$

$9.1(5.48)$

124 (42)

53 (18)

$89(30)$

$22.4(7.74)$

$64(22)$

88 (30)

$92(31)$

50 (17)

$222(75)$

128 (58)

147 (50)

119 (40)

28 (9)

266 (90)

155 (53)

66 (22)

64 (22)

11 (4) 


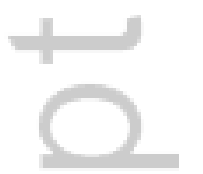

This article is protected by copyright. All rights reserved. 
Table 3. Comparisons between responses to measures of mental health, need and mental health service access and the single-item measure of self-reported mental health $(\mathrm{N}=294)$

\begin{tabular}{lllll}
\hline Variable & $\begin{array}{c}\text { Excellent/ } \\
\text { Very good } \\
\mathbf{n}(\boldsymbol{\%})\end{array}$ & Good & Fair & Poor \\
\hline Anxiety & $6(11)^{\mathrm{a}}$ & $34(31)^{\mathrm{b}}$ & $60(62)^{\mathrm{c}}$ & $24(80)^{\mathrm{c}}$ \\
Depression & $5(9)^{\mathrm{a}}$ & $33(30)^{\mathrm{b}}$ & $62(64)^{\mathrm{c}}$ & $28(93)^{\mathrm{d}}$ \\
Suicidality & $3(5)^{\mathrm{a}}$ & $17(15)^{\mathrm{a}, \mathrm{b}}$ & $28(29)^{\mathrm{b}, \mathrm{c}}$ & $16(53)^{\mathrm{c}}$ \\
Medication & $3(5)^{\mathrm{a}}$ & $22(20)^{\mathrm{a}, \mathrm{b}}$ & $29(30)^{\mathrm{b}}$ & $12(40)^{\mathrm{b}}$ \\
Perceived need & $22(39)^{\mathrm{a}}$ & $84(76)^{\mathrm{b}}$ & $86(89)^{\mathrm{b}, \mathrm{c}}$ & $30(100)^{\mathrm{c}}$ \\
Access & $13(59)^{\mathrm{a}}$ & $44(52)^{\mathrm{a}}$ & $54(63)^{\mathrm{a}}$ & $17(57)^{\mathrm{a}}$ \\
Previous mental & $13(23)^{\mathrm{a}}$ & $51(46)^{\mathrm{b}}$ & $69(71)^{\mathrm{c}}$ & $22(73)^{\mathrm{c}}$ \\
illness & & & &
\end{tabular}

Note: Clinically significant anxiety and depression. Subscript letters represent where column proportions differ significantly at the .05 level. Different subscript letters indicate statistical significance. 
Table 4. Preference for support activities $(n=285)$

\begin{tabular}{lc}
\hline Mental health support type & $\begin{array}{l}\text { Definitely } \\
\text { consider } \\
\mathrm{n}(\%)\end{array}$ \\
\hline Individual counselling/therapy & $187(66)$ \\
Guided relaxation programs & $141(49)$ \\
Education about mental health & $134(47)$ \\
Guided exercise programs & $132(46)$ \\
Relationship support & $92(32)$ \\
Peer support groups & $83(29)$ \\
Web-based individual therapy facilitated by a professional & $70(25)$ \\
Prescription psychotropic medication & $64(23)$ \\
Group-based therapy & $58(20)$ \\
\hline
\end{tabular}




\section{University Library}

\section{- M M N E R VA A gateway to Melbourne's research publications}

Minerva Access is the Institutional Repository of The University of Melbourne

Author/s:

Gilson, K-M;Davis, E;Johnson, S;Gains, J;Reddihough, D;Williams, K

Title:

Mental health care needs and preferences for mothers of children with a disability

Date:

2018-05-01

Citation:

Gilson, K. -M., Davis, E., Johnson, S., Gains, J., Reddihough, D. \& Williams, K. (2018).

Mental health care needs and preferences for mothers of children with a disability.

CHILD CARE HEALTH AND DEVELOPMENT, 44 (3), pp.384-391. https://doi.org/10.1111/ cch.12556.

Persistent Link:

http://hdl.handle.net/11343/283580 